\title{
An Astronomical Attempt to Determine the Temporal Origin of an Episode of the Armenian Epic "Sasnay Tsrer"
}

\author{
G. Broutian \\ NAS RA V. Ambartsumian Byurakan Astrophysical Observatory (BAO), Armenia
}

\begin{abstract}
An attempt was made to estimate the time period of the Armenian epic "Sasnay Tsrer" by astronomical means. An episode in which Tsovinar mentions a bright star as night guide for sending her sons from Mesopotamia to Armenia has been examined from the point of view of astronomy. On basis of the "khachapasht" (cross worshipers) self-proclamation of representatives of the House of Sasun and wearing the sign of the cross on their right hand, this guiding star is identified with the constellation Cygnus (ancient Armenian Angkh - Vulture), which depicts the outline of a cross in the northern sky. Subsequently, we calculated when Cygnus' main star, Deneb, was closest to the North Pole. The calculations show that this event took place 17,500 years ago. This time frame was taken as the time of the creation of the above-mentioned episode of the Sasnay Tsrer Epic.
\end{abstract}

Keywords: Epic Sasnay Tsrer: Astronomical dating: Angegh - Vulture - Cygnus constellation

\section{Introduction}

The temporal origin of creation of the Armenian Epic Sasnay Tsrer has been debated by various authors based on identifications of the heroes of the Epic and other historical individuals. For example, it is argued that real historical persons - Theodoros Rshtouni, lord of Taron and Sassoun Davit Bagratouni (Bagarat's son), lord of Sassoun Tornik Mamikonian, Yovnan form Khout and so on served as historical prototypes for characters of the Epic - K'eri Toros (Uncle Toros), David of Sassoun and Dzenov Ohan. ${ }^{1}$ According to these "identifications", Sasnay Tsrer dates back to the period of Arab invasions. $^{2}$

At the same time, there are references to Sanasar and his brother Baghdasar (in some versions under different names), one of the heroes of the first branch of the Epic, in the works of Movses Khorenatsi (V century) and Tovma Artsrouni (IX-X century). ${ }^{3}$ These facts date the time of the Epic to at least $\mathrm{V}$ century (in the time of Moses Khorenatsi).

However, these are not the only demarcations for the time of Sasnay Tsrer Epic. The two heroic brothers of the first branch of the Epic are clearly parallel in their activities and names to the sons of Sennacherib of Assyria (705-680 BC), Adrammelech and Sarasar (4 Kings, 19, 37). This connects time of the Epic to the era of the kingdom of Van. Of course, there is also temporal disagreement as according to the Epic, Sanasar and Baghdasar are contemporaries of the Caliph of Baghdad, whose character should be associated with the Arab invasions and the subsequent time periods. As such, there are clear timeline disagreements that are compounded by other factors forming bases to suggest

\footnotetext{
${ }^{1}$ Manouk Abeghian, Complete works, v. 1, Publ. of Academy of Sciences of RA, Yerevan, 1966 pp. 354 380, v. 8, 1985, pp. 1265 (in Armenian).

${ }^{2}$ It was on this basis that the 1000th anniversary of the Sasnay Tsrer Epic was celebrated in Soviet Armenia in 1939. See Armenian Soviet Encyclopedia, v. 10, pp. 195 - 199, Yerevan, 1984 (in Armenian).

${ }^{3}$ Movses Khorenatsi, History of Armenia, I, 23, p. 70-71, II, 07, p. 111, with diligence of Manouk Abeghian, Tbilisi, 1913 (in Armenian), Tovma Artsrouni, and Anonym, The history of the House of Artsrouni, Yerevan University publ., Yerevan, 1978, p. 78 (in Armenian).
} 
older time periods for the origins of this Epic. These examples demonstrate that the time periods obtained by identi

cation of various epic characters with a known historical individual can vary greatly. Identification and combination of characters with coincidence of names or certain actions of historical figures known to us cannot be relied upon. Therefore, it is not accurate, to determine the time of the Sasnay Tsrer Epic or some of its episodes by identifying the names of the heroes. As such, it is necessary to search for other means to determine the time of the whole Epic or some of its episodes.

\subsection{The problem}

It is known that the most reliable way to determine time of different historical events is to connect the events with an astronomical phenomena (e.g., solar and lunar eclipses, nova and supernova airs, appearance of some comets, etc.) and approximate date of the event through this approach. The same approach can be used for literary and folklore compositions, if it is possible to find references to various astronomical phenomena in the narrative. Now we will explore the possibility to determine origins of Sasnay Tsrer by astronomical means. Fortunately, narratives that have come down to us in this Epic have preserved a number of fragments that, when properly interpreted astronomically, can serve as evidence for timing of the formation of those fragments. Ignoring enumeration of the examples, we will focus on discussion of one of them. In Sasnay Tsrer Epic, from the beginning of the first branch, definition of the Armenian nation as a "khachapasht" (worshiper of the cross) is repeated several times in different narratives. ${ }^{4}$ In addition, all the main heroes of the House of Sasoun carry on their right arm a special sign given from the Heaven - the Cross. ${ }^{5}$ This is mentioned in different narratives as "Khach Patrastin (Cross Ready)", "Paterazmin (Cross of War)", "Pataragin (Cross of Liturgy)", "Khach (Meat Cross)" etc. Moreover, the first bearer of this miraculous cross - Sanasar, receives it from Heaven after bathing in the milky spring, and getting stronger and fully armed.

"Angels put the Cross of War

On the right arm,

So that no beats could harm Sanasar". ${ }^{6}$

This is noteworthy as there is a large constellation in the northern hemisphere, that forms a huge brilliant cross with its 5-6 main bright stars in northern sky. This concerns to the Swan (Cygnus) constellation, that forms the heavenly cross with the stars: $\alpha$ Cygni (Deneb), $\beta$ Cygni (Albireo), $\gamma$ Cygni (Sadr), $\delta$ Cygni (Fawaris), $\varepsilon$ Cygni (Aljanah), and $\eta$ Cygni. On modern heavenly charts the Swan constellation is located between the northern constellations of Draco, Lyra, Vulpecula, Pegasus, Cepheus and Lacerta (see Figure 1).

We also know that according to ancient Armenian division of the starry sky this constellation was called Ang l (or Ank 1), that means "Vulture". There is an image of a vulture instead of a swan on an Armenian heavenly chart printed by Ghoukas Vanandetsi in 1695 in Amsterdam (Figure 2). Near the tail of the bird we have a legend "Ankh" which means "Vulture". Besides, names of constellations (and individual stars) stated in Armenian medieval manuscript sources do not contain the name "Swan", however, there is mention of the name "Kerkez", which means "vulture".?

It is known that the bird vulture was the heavenly character of the ancient god Nergal. ${ }^{8}$ Nergal was the main god of Hayasa - one of the oldest Armenian states (BC XIV - XIII c). ${ }^{9}$ Thus, the entitlement

\footnotetext{
${ }^{4}$ See for example, Sasountsi Davit, Publ. of Academy of Sciences of RA, Yerevan, 1961, pp. 4, 5, 23, 48. (in Armenian).

${ }^{5}$ Ibid. pp. 40, 41.

${ }^{6} \mathrm{Ibid}$

${ }^{7}$ See, for example, Grigor Broutian, Armenian Calendar, Mother See of Holy Etchmiadzin, 1997, page 490 (in Armenian). Not all of the names of stars and constellations listed here have been identified with modern-day celestial realities, but some may have been identified as reliable. For Kerkez, Acharian gives: There is a Persian word karkas (kerkes) "Vulture" (Hr. Acharian, Armenian Etymological Dictionary, vol. 4, Yerevan University Press, Yerevan, 1979, p. 574).

${ }^{8}$ Armen Davtian, Armenian stellar mythology, Yerevan, 2004, pp. 207-222 (in Armenian).

${ }^{9}$ Gr. Khapancian, Historical-linguistic studies, Publ. of Academy of Sciences of Arm. SSR, Yerevan, 1956, pp. 88-89 (in Russian).
} 


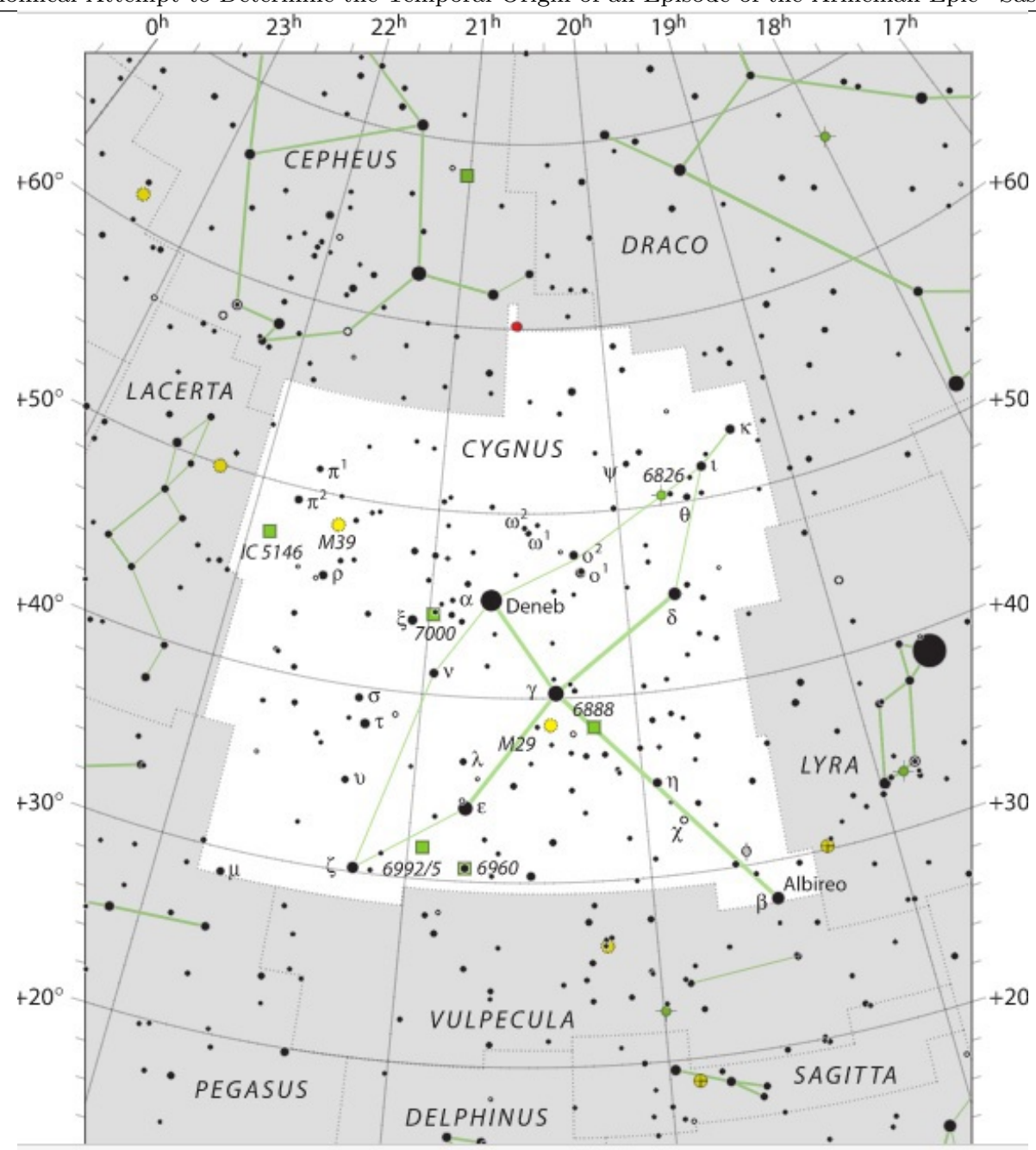

Figure 1. The Armenian Ang 1 - Vulture (modern Swan - Cygnus) constellation and its heavenly neighborhood on modern sky chart

"khachapasht" (worshiper of the cross) means a person who worships the god Angl - Nergal. ${ }^{10}$ On the other hand, we know that worshiping any god is essentially the same as worshiping that celestial image of that god. Taking into account the fact that Hayasa was only one of the ancient Armenian states, we get that "khachapasht" = worshiper of Vulture - Nergal = resident of Hayasa = Armenian. In other words, we got exactly what is directly written in the Epic.

Now let's look at how it is possible to use this identification (Armenian $=$ worshiper of cross $=$ worshiper of Nergal = worshiper of the Swan-Vulture star) to determine the origin of the Epic Sasnay Tsrer.

Of course, the above-mentioned relationship alone is not sufficient to determine the origin of the Epic. We need additional information to help us estimate the correct time of the relationship. Details in the following episode of the Epic can provide the additional information needed. After Tsovinar learns that the Caliph has promised to sacrifice her sons to his Great Idol, she orders them to flee to Armenia to their grandfather to the north.

"Run away, go to the city of the Armenian king.

\footnotetext{
${ }^{10}$ This is also shown by the carrying of a special (bestowed from heaven) cross on the right arm of the heroes of the House of Sasoun. They receive the cross after special (ritual) actions (to pray, to bathe in a special spring). In other words, it turns out that they receive the sign of this cross after a special offering ritual. This is reminiscent of becoming a mahtesi (a person who had gone as a pilgrim to Jerusalem) highly valued in Christianity (especially among Armenian Christians), which was traditionally associated with making a covenant in Jerusalem (Christ's tomb) and then having a special (including a special cross) tattoo on the arm. In fact, the cross on the arm of the heroes of the House of Sasoun, is the expression of the celestial symbol of the god worshiped by the cross worshiping nation on their own body.
} 


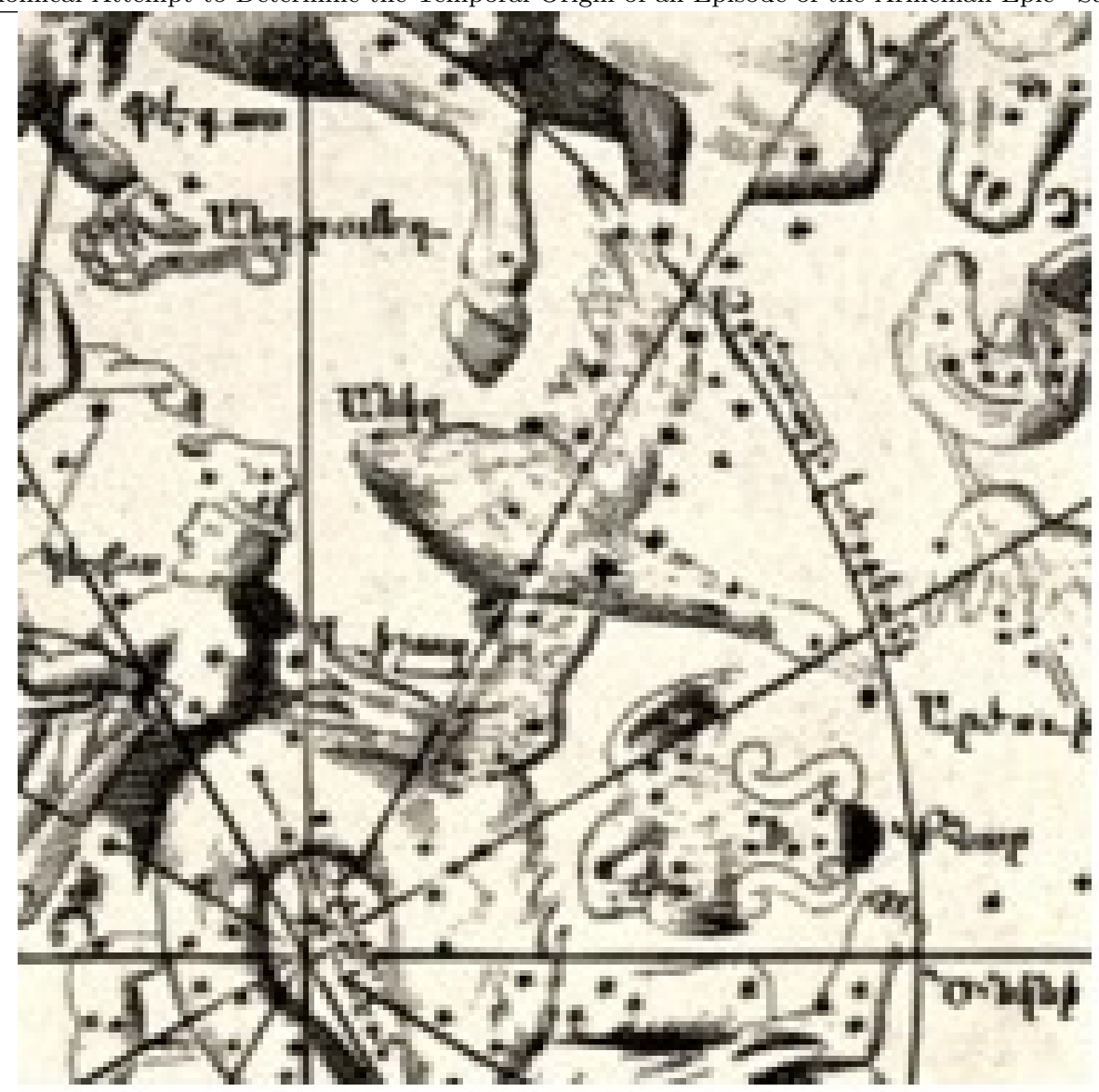

Figure 2. The Armenian Ang 1 (modern Swan - Cygnus) constellation and the neighboring constellations on the heavenly chart printed in 1695 in Amsterdam by Ghoukas Vanandetsi

Catch the bright star at night as a target." 11

In other words, as a night guide to the journey, she points to a bright star in the sky and orders the sons to go in the direction of that star, confident that it will take them to Armenia.

Here we have a combination of several realities!

1) Tsovinar is instructing her cross worshiper children to always follow the same star.

2) That star is bright.

3) She assures that guided by that star, her sons will reach their homeland, Armenia.

4) To be guided by a star implies following the deity whose heavenly mythical image is that star, to worship that god and to do the will of that god.

5) In order to reach Armenia from the territory of Baghdad (i.e., from central Mesopotamia) guided by a star, it is necessary for that star to be in the northern part of the sky, as Armenia is located north of Mesopotamia.

6) As a result of the Earth's daily rotation, all the stars in the sky revolve around the pole once a day and no star has a stable position in the sky. During the night, the stars move in the sky from east to west with a speed of one rotation in 24 hours. Therefore, the same star at different times of the night has different positions in the sky. The position of the star in the night sky can change from the eastern horizon to the western horizon. Therefore, it is impossible to point out any star the direction of which will always lead to a specific destination including Armenia. Due to its position, the only star in the sky that can be used as a guide at night, is the polar 
An Astronomical Attempt to Determine the Temporal Origin of an Episode of the Armenian Epic "Sasnay Tsrer"

star. That is, only the star at the north pole of the sky, or very close to the pole, can serve as a guide. ${ }^{12}$ Since focus of events is in the northern hemisphere of the Earth, the star near the north pole (or at the very pole) can play that role in the sky. In other words, we conclude that the only star that can serve as a guide in Earth's northern hemisphere is the star at or near the North Pole.

Meanwhile, there is an inconvenience here. In the epic it is clearly mentioned that the star, which is the guide of the night, is "bright". The instruction given to the sons has a certain mention: "Catch the bright star as a target." Today the star in the night sky, which is very close to the North Pole, called "Polaris", is not so bright. This star is known as the F7Ib class yellow supergiant star, located at a distance of 433 light-years from us, with an average magnitude of $1^{m} \cdot 98 .^{13}$ That is, it is approximately a second magnitude star. In order to be designated "bright", the star had to be at least of $1.0^{m}$ - $1.5^{m}$ magnitude or brighter. According to modern data, the Polar Star is the 48th in the list of the brightest stars (excluding the Sun). That is, there are 47 stars in the night sky brighter than Polaris. Along with this, let us remember that as a result of the Earth's precession, the direction of the Earth's polar axis changes over the millennia, and thus the appearance of the night sky. This recurring phenomena was discovered in the 2nd century BC by Hipparchus. According to current adjusted data, its periodicity is 25,776 years. At this rate, the axis of daily rotation of the $\operatorname{Earth}^{14}$ forms a cone around the axis of the ecliptic at an angle of about 47 degrees. Naturally, during that time the real pole can "appear" near different stars. Naturally, during that time the real pole can "appear" near different stars. At present, the north pole of the sky (the pole of the world) is right next to the star called the Polaris (the angular distance is $00^{\circ} 44^{\prime} 09^{\prime \prime}$ and in different historical eras it has been close with different stars. For example, 6,000 years ago, the north pole was near the star $\alpha$ Draconis (Tuban), 12,500 years ago it was near the star $\alpha$ Lyrae (Vega), which is the brightest star in the northern hemisphere.

Modern calculations show that during these long-term "wandering" of the pole, about 18,000 years ago, the north pole of the sky was near the star currently known as $\alpha$ Cygni (Deneb). In the central part of the Figure 3 there is the pole of ecliptic in the constellation of the Dragon. The position of the north pole of the world in different eras is presented as a circle around it. The current $+2,000$ year is at the top. To the right of it (clockwise) are the past dates up to 10,000 BC, and to the left (counterclockwise) are given the dates of the future, up to $+14,000$. The figure clearly shows that about 18,000 years ago, the North Pole was right next to the main star of the constellation Cygnus (Swan, formerly - Vulture). We also know that Deneb star is a variable star of class A2Ia with an average visible stellar magnitude of $1 \mathrm{~m} .25$ and is approximately a first magnitude blue-white star. A simple calculation shows that Deneb is about twice as bright as the Polaris (exactly 1.9589 times) and in the list of the brightest stars in the sky, Deneb is in the 20th place (versus of the 48th place for Polaris).

Let's add another important fact to our discussion. We know that the Swan (Vulture) constellation has played an important role in ancient Armenia, as witnessed by the ancient megalithic monument known as "Zorats K'arer" in Syunik region of Armenia near the town Sisian. This megalithic structure is remarkable in several respects. First is the arrangement of megaliths that quite accurately replicate the arrangement of the bright stars of the Swan (Vulture) constellation in the sky. Second is that according to latest research, arrangement of the megaliths of this monument best correspond to arrangement of stars of the constellation Swan, which existed 32,000 years ago (Malkhasyan, 2020). In other words, at least a certain part of the Zorats Karer monument must have been erected 32,000 years before us and there must have been the cult of the Vulture in Armenia which appears in the form of a celestial cross, the expression of which is the modern Swan constellation.

\footnotetext{
${ }^{12}$ What is said here refers to the territory of the northern hemisphere of the Earth. For the area of the southern hemisphere, the star at or near the south pole plays the same role.

${ }^{13}$ Stellar magnitude is a quantitative measure of the luminosity of stars. It was first used by Hipparchus in the 2nd century BC, dividing the observed brightness scale of the stars into 6 groups. According to Hipparchus, the brightest stars were considered to be of the first magnitude, and the faintest stars were of the sixth magnitude.

${ }^{14}$ In professional astronomical literature, this is also called the "axis of the world", and the points of intersection of this axis with the celestial sphere are called "poles of the world" (north and south, respectively).
} 


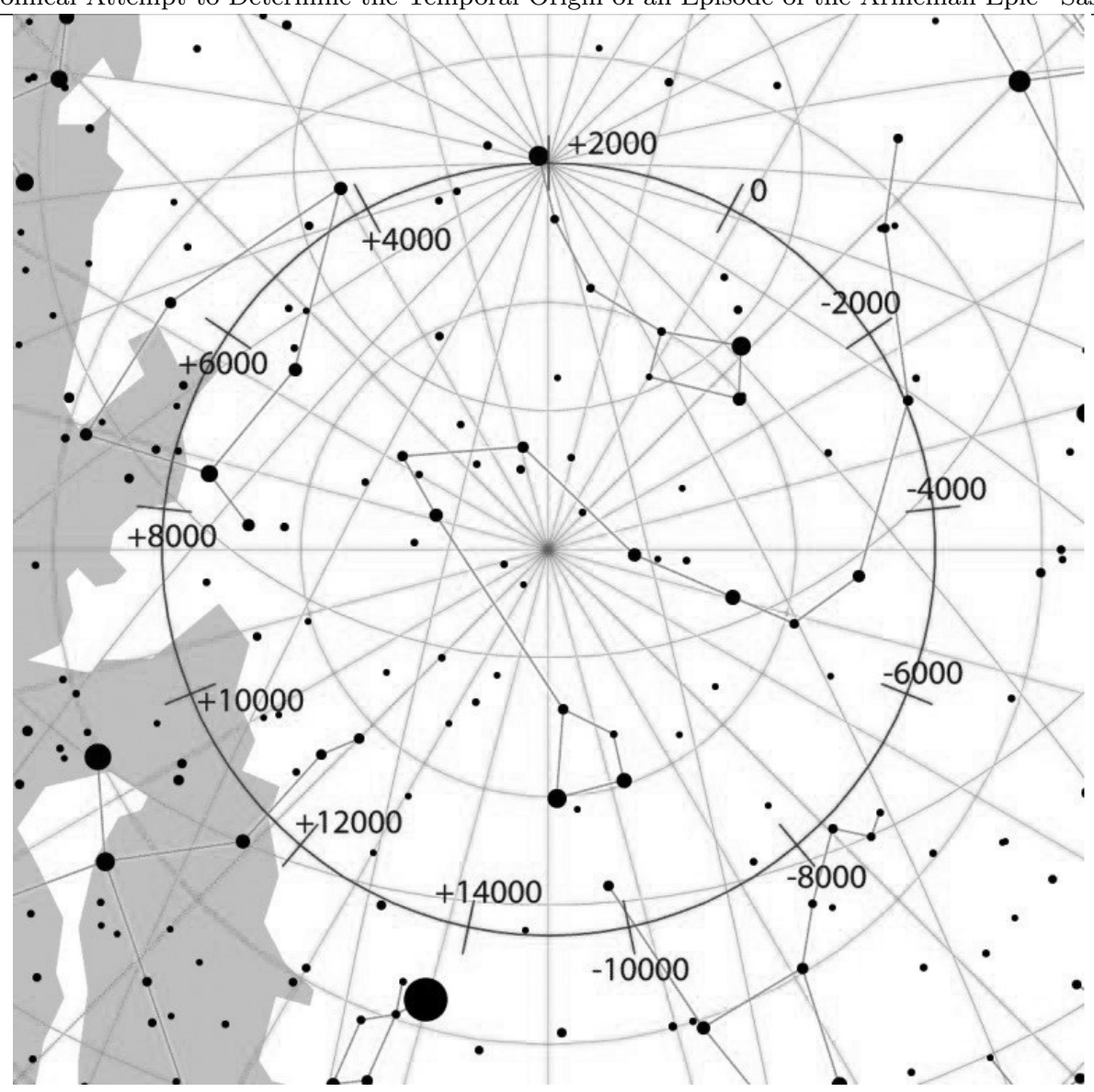

Figure 3. The change in the position of the north pole of the sky during one period of precession (the pole of the ecliptic is in the center). Above is the picture of the star Polaris in the constellation Ursa Minor (Little Bear, old Armenia - The Other Chart), and below the bright star is the Lyrae star Vega. On the left side is the constellation Swan with the stars Deneb, Sadr, Favaris and Albireo.

In this regard, we can also mention the T-shaped pillar with the image of a vulture found in the ancient site of Portasar (Göbekly tepe in Turkish), not far from the city of Edessa (Urfa) in the southern part of historical Armenia. The vulture depicted on this pillar also corresponds to the arrangement of the stones of the megalithic part of the Zorats K'arer and the arrangement of the stars of the constellation Swan (Cygnus). ${ }^{15}$

Now, let's aggregate everything that relates to the "cross worshipper" nature of Armenians.

a. Armenians considered themselves "cross worshipper"

b. Stars forming the image of a cross comprise the constellation Vulture = Swan - Cygnus

c. The god Angl = Nergal was also the main deity of Hayasa, one of the oldest Armenian state formations

d. Main heroes of the House of Sasoun wore on their right arm a special sign of cross bestowed from Heaven, that is, the image of the Vulture-Swan constellation

e. For those traveling north from Mesopotamia to Armenia, only the star at the North Pole or one very close to the pole could be a guide in the night sky.

Taking all this into account, we conclude that Tsovinar should have instructed her sons to follow as a guiding star in the night sky, the main star of the constellation Vulture-Swan, which is twice as bright as the relatively weak star called Polaris. Hence, the episode describing the departure of Sanasar and Baghdasar from Mesopotamia to Armenia must have been created when the main star of the Vulture-Swan was as close as possible to the north pole of the sky.

This is how we get an alternative way to determine the creation period of the episode of Sasnay

\footnotetext{
${ }^{15}$ Vachagan Vahradian, Marine Vahradian, On the name of the monument K'arahounj, "Bazmavep", 2010, 1-2, pp.161177. (In Armenian). In particular see Figure 5, p. 176.
} 
Tsrer. In other words, it turns out that at least a certain part of the Sasnay Tsrer Epic must have been created at the time when the north pole of the sky was near the main star of the Vulture-Swan constellation Deneb. Thus, in order to find the time of the creation of this episode in the Sasnay Tsrer Epic, it is necessary to find out in what era the north pole of the sky was near the star Deneb.

The diagram in Figure 3 clearly shows that the north pole near Deneb was about 16,000 years before birth of Christ, which means 18,000 years ago. Of course, determining the time on this diagram visually can contain a large level of error. On the graph, both the position of the stars and the path of the North Pole are given approximately. In order to have the most reliable result, it is necessary to perform the calculations using the formulas of spherical trigonometry.

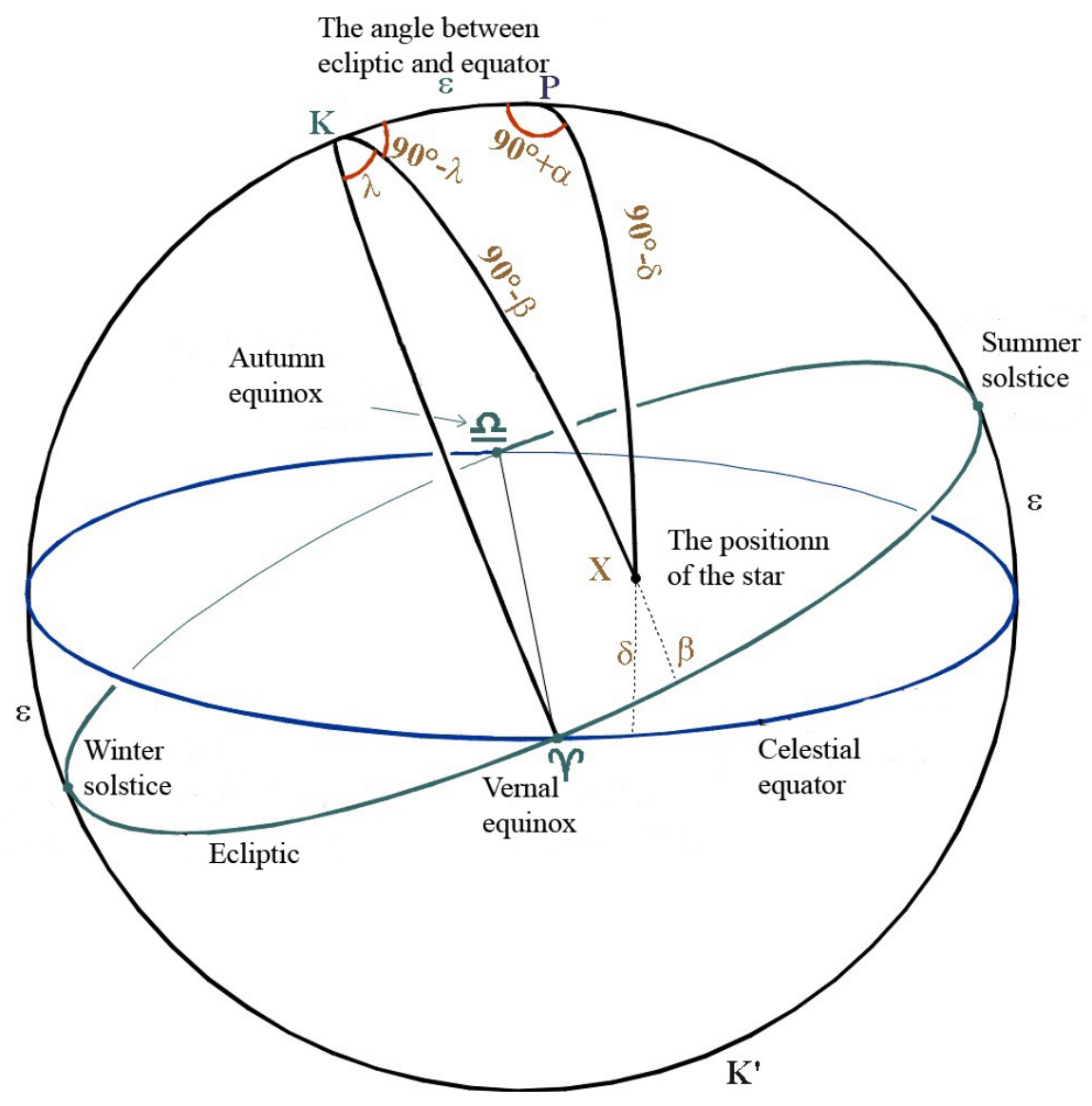

Figure 4. The relation of ecliptic and equatorial coordinates. P denotes the north pole of the world, $\mathrm{K}$ denotes the north pole of the ecliptic, and $\mathrm{X}$ denotes the position of the star in the sky. $\alpha$ and $\delta$ are the equatorial coordinates of the star (right ascension and declination), and $\beta$ and $\lambda$ are the equatorial coordinates (latitude and longitude).

\section{The Calculations}

As the north pole of sky (pole of the world), as a result of the centuries-old precession of the Earth's polar axis, revolves around the north pole of the ecliptic with a period of 25,776 years, the star that we are interested in will be in the position closest to the north pole of the sky when the north pole of the ecliptic, the north pole of the sky and the star are arranged on a line. Specifically, on the same arc of the great circle of the celestial sphere (Figure 4). To solve our problem, let us consider the spherical triangle KPX on the celestial sphere. The diagram in Figure 4 shows that the condition, when the ecliptic pole, the north pole of the sky and the star we are interested in are arranged on a line is equivalent to the condition that angle $\mathrm{K}$ in the triangle KPX (angle XKP) is equal to 0. That is the change in the position of the north pole of the sky during one period of precession (the pole of the ecliptic is in the center). Above is the picture of the star Polaris in the constellation Ursa Minor 
(Little Bear, old Armenia - The Other Chart), and below the bright star is the $\alpha$ Lyrae star - Vega. On the left side is the constellation Swan with the stars Deneb, Sadr, Favaris and Albireo.

$$
90^{\circ}-\lambda=0
$$

From above:

$$
\lambda=90^{\circ}
$$

Using formulas from the spherical trigonometry, we move from equatorial and coordinates of the star to its ecliptic coordinates $\beta$ and $\gamma$.

$$
\begin{gathered}
\tan \lambda=\frac{\sin \alpha \cos \varepsilon+\tan \delta \sin \varepsilon}{\cos \alpha} \\
\sin \beta=\cos \varepsilon \sin \delta-\sin \varepsilon \cos \delta \sin \alpha
\end{gathered}
$$

Here is the angle between the ecliptic and the equator. From here we get for $\gamma$ and $\beta$.

$$
\begin{gathered}
\lambda=\left\{\begin{array}{cc}
\arctan \frac{\sin \alpha \cos \varepsilon+\tan \delta \sin \varepsilon}{\cos \alpha}, & \cos \alpha>0, \sin \alpha \cos \varepsilon+\tan \delta \sin \delta \sin \varepsilon \geq 0 ; \\
\arctan \frac{\sin \alpha \cos \varepsilon+\tan \delta \sin \varepsilon}{\cos \alpha}+360^{\circ}, & \cos \alpha>0, \sin \alpha \cos \varepsilon+\tan \delta \sin \varepsilon<0 \\
\arctan \frac{\sin \alpha \cos \varepsilon \tan \delta \sin \varepsilon}{\cos \alpha}+180^{\circ}, & \cos \alpha>0 .
\end{array}\right. \\
\beta=\arcsin (\cos \varepsilon \sin \delta-\sin \varepsilon \cos \delta \sin \alpha)
\end{gathered}
$$

By inserting into the equation 3 the values of equatorial coordinates $\alpha$ and $\delta$ of Deneb $\left(\alpha_{2000}\right.$ $=20^{h} 41^{m} 25.9^{s}=310.3579166^{\circ} ;$ and $\left.\delta_{2000}=45^{\circ} 16^{\prime} 49^{\prime \prime}=45.2802777^{\circ}\right)$ and the adjusted value of $\epsilon$ $\left(23^{\circ} 26.21=23.4391666^{\circ}\right)$, we get its ecliptic coordinates $\beta$ and $\lambda$.

In our case $\cos \alpha>0$, and $\sin \alpha \cos \epsilon+\operatorname{tg} \delta \sin \epsilon<0$, then the second of the conditions of equation 3 occurs, so we add $360^{\circ}$ to the value obtained for $\lambda$.

For the ecliptic longitude of Deneb we obtain $\lambda=335.3315^{\circ}$. If we denote the ecliptic longitude $\lambda$ of Deneb at the position closest to the North Pole by $\lambda_{0}$, then we can write the above condition as follows: $\lambda_{0}=90^{\circ}$. Hence: $\lambda-\lambda_{0}=335.3315^{\circ}-90^{\circ}=245.3315^{\circ}$.

The ecliptic latitude is not dependent on time, and from equation 4 we get $\beta=59.90676344^{\circ}$ or $\beta=59^{\circ} 54^{\prime} 24.35^{\prime \prime}$

The rest is simple arithmetic. Assuming that the Earth's polar axis rotates at a constant speed ${ }^{16}$, we calculate how many years it will take the rotation of this axis at an angle of $245.3315^{\circ}$ if the complete rotation takes 25,776 years. That is equal to:

$$
\frac{245.3315 \cdot 25776}{360}=17565.7654
$$

It turns out that the star Deneb was in the closest position to the north pole of the sky $17,565.7684$ years $\operatorname{ago}^{17}$. The fractional part of this number is not significant in the case of our problem as the magnitude of the error for determining the time period is significantly greater than the impact of the fractional value. To evaluate the possible error in determining time with this method, consider that Deneb can be considered at the North Pole when looking at the sky with the naked eye, it is "forward" or "back" from its nearest position by no more than its angular distance from the pole. From the above $\beta=59.90676344^{\circ}$ we get that Deneb was at the closest position to the pole at a distance of $90^{\circ}-59.90676344^{\circ}-23.440833^{\circ}=6.65240356^{\circ}$. This is equivalent to an angle of $6^{\circ} 39^{\prime} 8.7^{\prime \prime}$. This value is less than the angular distance between Deneb and Sadr. It is of this order that the error in the observational determination of the position of the North Pole should be. This type of displacement of the pole takes place in about 500 years (exactly 476 years). As such, the error of the determination of the time period is 500 years with the fluctuation interval on the order of 1000 years.

\footnotetext{
${ }^{16}$ The latest data show that we can consider the speed of this precession to be constant.

${ }^{17}$ It is noteworthy that at that time the north pole almost coincided with the stars o1 Cygni and o2 Cygni representing the celestial vulture's beak.
} 
Summarizing the calculation results, we determine that the episode in Sasnay Tsrer Epic about Tsovinar sending his two twin sons from Baghdad to Armenia could have been created about 17,500 years ago (with 1000 years of uncertainty). Of course, this finding does not suggest that the whole Epic was created at that time. The result obtained refers only to the specific subject matter that the episode discussed from Sasnay Tsrer was created 17,500 years ago.

In order to determine creation time of the whole Epic, it is necessary to examine other episodes that contain astronomical relationships. In this manner, it can be possible to determine time period of the creation of different episodes and different parts of this Epic. Without a doubt, this will be very beneficial for the complete and correct understanding of Sasnay Tsrer.

\section{References}

Malkhasyan H. A., 2020, Communications of the Byurakan Astrophysical Observatory, 67, 27 